\title{
Nontuberculous mycobacteria
}

\section{Les mycobactéries non tuberculeuses}

In the current issue of the Canadian Respiratory Journal, Al-Houqani et al (1) (pages 19-24) examined the isolation prevalence of nontuberculous mycobacteria (NTM) in Ontario in 2007, and compared it with previous experience. Isolation prevalence essentially refers to the number of positive sputum cultures for these organisms related to the number of patients and the population at large. The data were from the Toronto Public Health Laboratory (Toronto, Ontario), which processes at least $90 \%$ of these sputum specimens for the province. The same group previously examined the same question over the years 1997 to 2003, and found that the prevalence increased from approximately nine patients per 100,000 population to 14 patients per 100,000 population (2). In 2007, it increased to approximately 19 patients per 100,000 population, which, needless to say, was highly significant.

What is going on here? Changes in isolation techniques did not occur during either study period $(1,2)$. Either colonization and/or disease due to these organisms were, in fact, increasing, or physicians were finding more of them without such an increase. If NTM disease/colonization were not increasing, but physicians were requesting more cultures based on general awareness, the increase in isolation prevalence should have been accompanied by an increase in negative cultures, but this did not occur. Indeed, the fraction of samples that were positive - approximately $10 \%$ - did not change between 2002 and 2007. Thus, given the same set of patients, the findings cannot be based on physicians simply ordering more tests. The increase may also be due to differing patterns of behaviour by physicians in following patients. Positive cultures must represent both incidence and prevalence and, if the former did not change, but more physicians obtained more sputum samples during follow-up, then isolation prevalence would increase. Unfortunately, data for the years between 2003 and 2007 were not available; therefore, the frequency of repeat offenders could not be measured. The authors discount this because before 2003, it was uncommon for one patient to have positive cultures in more than one year, but admit that this may not be currently relevant. On the other hand, it may be that physicians improved their discriminatory powers and, consequently, discovered more cases. The obvious cause for this may be an increased frequency of computed tomography lung scans yielding patterns similar to the 'tree and bud' suggestive of NTM infection. This, it seems to me, is a reasonable hypothesis, but there is nothing in the data from Al-Houqani et al that suggests that this, in fact, occurred; the frequency of positive sputa did not increase as it perhaps should have with improved methods of detection.

The isolation prevalence of NTM of 19 patients per 100,000 population in Ontario was higher than reported elsewhere, but others have shown that it is increasing, although the other results are not as current as those of Al-Houqani et al. What does this mean in terms of disease burden? As implied above, isolation of these organisms does not necessarily signify illness because it could result either from colonization without disease or, possibly, contamination with ubiquitous organisms. Smear-positive sputa have been associated with disease, and the frequency of positive smears remained constant in Ontario, implying an increase in this index of disease. Previously, the same group estimated that approximately $25 \%$ of patients with positive cultures have disease - also implying an increase (3). Al-Houqani et al believe that disease due to these organisms is likely increasing, led by an increase in the isolation of avian mycobacteria. The reasons for such an increase are unclear, and may relate to the increasing prevalence of
Dans le présent numéro de la Revue canadienne de Dneumologie, Al-Houqani et coll. (1) (pages 19-24) ont examiné la prévalence d'isolement des mycobactéries non tuberculeuses (MNT) en Ontario en 2007 et l'ont comparée à une expérience antérieure. Au fond, la prévalence d'isolement désigne le nombre de cultures positives d'expectorations pour ces organismes, par rapport au nombre de patients et à l'ensemble de la population. Les données étaient tirées du Toronto Public Health Laboratory (de Toronto, en Ontario), qui ont traité au moins $90 \%$ de ces échantillons d'expectorations pour la province. Le même groupe a déjà étudié la même question entre 1997 et 2003 et a déterminé que la prévalence était passée d'environ neuf patients pour 100000 habitants à 14 patients pour 100000 habitants (2). En 2007, elle est passée à environ 19 patients pour 100000 habitants, ce qui, il va sans dire, est hautement significatif.

Que se passe-t-il? Il n'y a pas eu de modifications aux techniques d'isolement pendant les deux périodes d'étude $(1,2)$. En fait, seules la colonisation ou les maladies causées par ces organismes augmentaient, ou les médecins en trouvaient davantage sans qu'il y ait d'augmentation de ce type. Si les maladies ou la colonisation par des MNT n'augmentaient pas, mais que les médecins demandaient plus de cultures en raison d'une sensibilisation généralisée, la hausse de la prévalence d'isolement aurait dû s'accompagner d'une augmentation des cultures négatives, mais cette situation ne s'est pas vérifiée. En fait, la fraction d'échantillons positifs, environ $10 \%$, n'a pas changé entre 2002 et 2007. Ainsi, compte tenu du même groupe de patients, les observations ne peuvent pas se fonder sur la simple demande d'un plus grand nombre de tests. L'augmentation peut aussi être attribuable à divers modes de comportement par les médecins dans le suivi des patients. Les cultures positives doivent représenter à la fois l'incidence et la prévalence et, si l'incidence n'a pas changé, mais que plus de médecins ont obtenu un plus grand nombre d'échantillons d'expectorations pendant le suivi, la prévalence d'isolement serait plus élevée. Malheureusement, les données portant sur les années 2003 à 2007 n'étaient pas accessibles. Par conséquent, il était impossible de mesurer la fréquence de récidivistes. Les chercheurs ont ignoré ce facteur parce qu'avant 2003, il était peu courant pour un patient d'avoir des cultures positives plus d'un an, mais ils admettent que cette observation n'est peut-être plus pertinente. Par contre, il se peut que les médecins aient amélioré leurs pouvoirs discriminatoires et, par conséquent, aient découvert plus de cas. La cause évidente peut être une fréquence accrue de tomodensitométries pulmonaires présentant des motifs similaires à "l'arbre en bourgeons ", évocateur des infections à MNT. Selon moi, c'est une hypothèse raisonnable, mais rien dans les données d'Al-Houqani et coll. n'indique que c'est bien ce qui s'est produit. La fréquence d'expectorations positives n'a pas augmenté, ce qui devrait peut-être être le cas grâce à l'amélioration des méthodes de détection.

La prévalence d'isolement des MNT chez 19 patients pour 100000 habitants en Ontario était plus élevée qu'ailleurs, mais d'autres ont démontré qu'elle augmente, même si ces résultats ne sont pas aussi à jour que ceux d'Al-Houqani et coll. Qu'est-ce que ça veut dire pour ce qui est du fardeau de la maladie? Comme on l'a indiqué ci-dessus, l'isolement de cet organisme ne se traduit pas nécessairement par une maladie parce qu'il peut résulter soit d'une colonisation sans maladie, soit, peut-être, d'une contamination par des organismes omniprésents. Les frottis d'expectoration positifs s'associent à la maladie, et la fréquence de frottis 
immunocompromised hosts - something that cannot currently be measured. Regardless of the reason, an increase is bad news. Disease due to these organisms is difficult to ascertain with certainty and very difficult to treat (4). Currently, management of individual patients with NTM is much more difficult than management of pulmonary tuberculosis.

\section{REFERENCES}

1. Al-Houqani M, Jamieson F, Chedore P, Mehta M, May K, Marras MK. Isolation prevalence of pulmonary nontuberculous mycobacteria in Ontario in 2007. Can Respir J 2011;18:19-24.

2. Marras TK, Chedore P, Ying AM, Jamieson F. Isolation prevalence of pulmonary non-tuberculous mycobacteria in Ontario 1997-2003. Thorax 2007;62:661-6.

3. Marras TK, Daley CL. Epidemiology of human pulmonary infection with non-tuberculous mycobacteria. Clin Chest Med 2002;23:553-67.

4. Griffith DE, Aksamit T, Brown-Elliott B, et al. An official ATS/IDSA statement: Diagnosis, treatment and prevention of nontuberculous mycobacteria disease. Am J Respir Crit Care Med 2007;175:367-416. positifs est demeurée constante en Ontario, ce qui laisse croire à une augmentation au sein de cet indice de maladie. Par le passé, le même groupe estimait qu'environ $25 \%$ des patients ayant une culture positive étaient atteints d'une maladie, ce qui sous-tend également une augmentation (3). Al-Houqani et coll. croient que la maladie causée par cet organisme est probablement en croissance, compte tenu de l'augmentation de l'isolement des mycobactéries aviaires. Les raisons d'une telle augmentation ne sont pas claires et pourraient être liées à la prévalence croissante d'hôtes immunodéprimés, ce qui ne peut être mesuré pour l'instant. Quelle que soit la raison, une augmentation signifie une mauvaise nouvelle. Les maladies causées par ces organismes sont difficiles à déterminer avec certitude et sont très difficiles à traiter (4). En ce moment, la prise en charge des patients atteints de MNT est beaucoup plus difficile que la prise en charge de ceux atteints de tuberculose pulmonaire.

\section{RÉFÉRENCES}

1. Al-Houqani M, Jamieson F, Chedore P, Mehta M, May K, Marras MK. Isolation prevalence of pulmonary nontuberculous Mycobacteria in Ontario in 2007. Can Respir J 2011;18:19-24.

2. Marras TK, Chedore P, Ying AM, Jamieson F. Isolation prevalence of pulmonary non-tuberculous mycobacteria in Ontario 1997-2003. Thorax 2007;62:661-6.

3. Marras TK, Daley CL. Epidemiology of human pulmonary infection with non-tuberculous mycobacteria. Clin Chest Med 2002;23:553-67.

4. Griffith DE, Aksamit T, Brown-Elliott B et coll. An official ATS/IDSA statement: Diagnosis, treatment and prevention of nontuberculous mycobacteria disease. Am J Respir Crit Care Med 2007;175:367-416. 


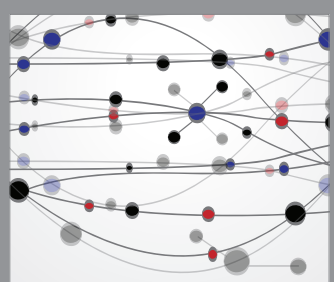

The Scientific World Journal
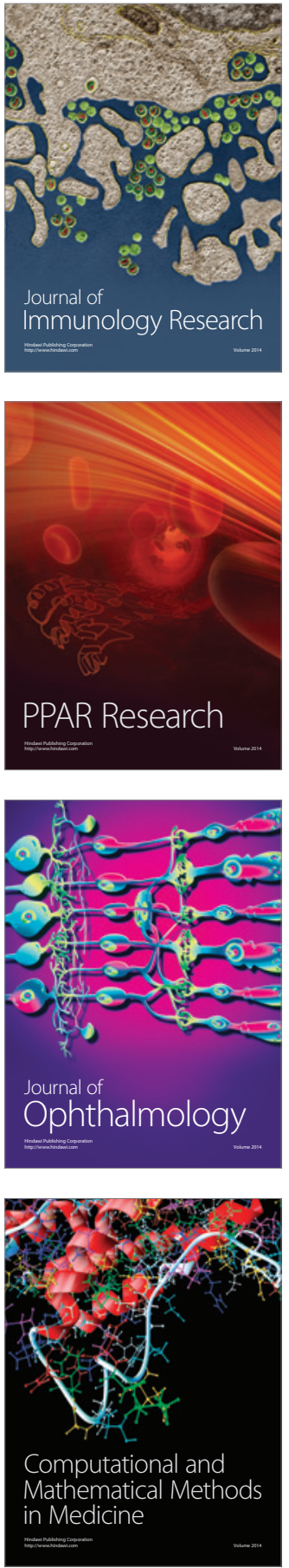

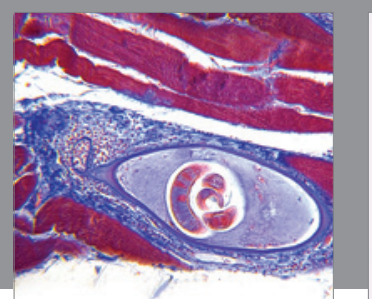

Gastroenterology Research and Practice

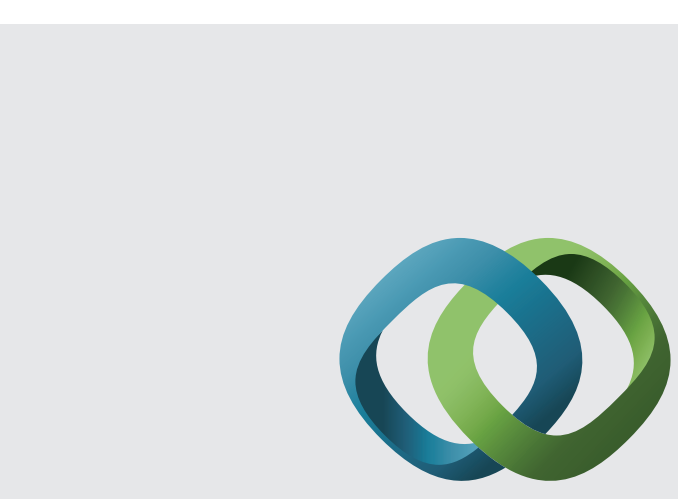

\section{Hindawi}

Submit your manuscripts at

http://www.hindawi.com
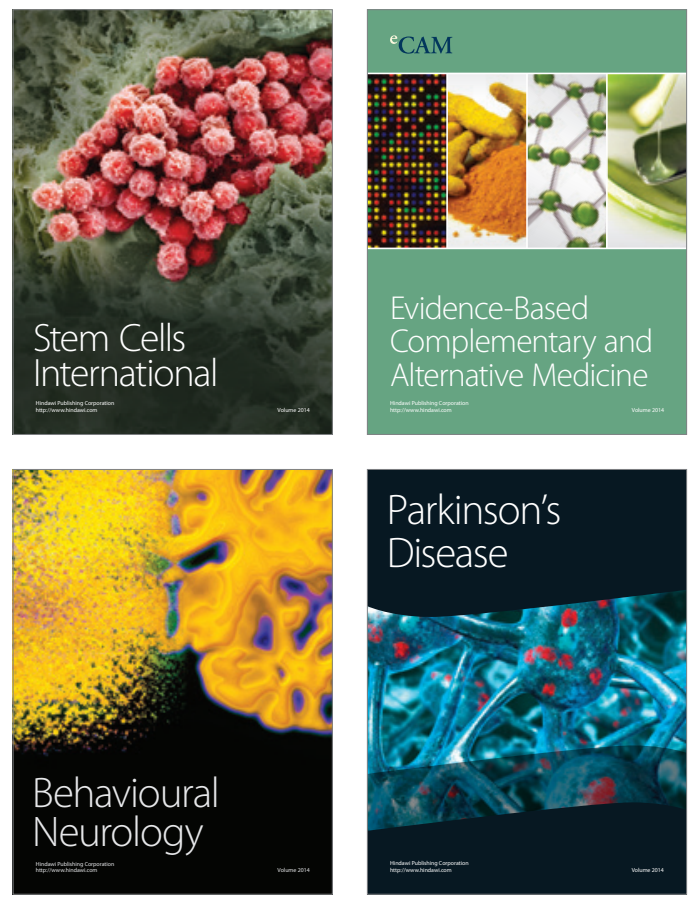
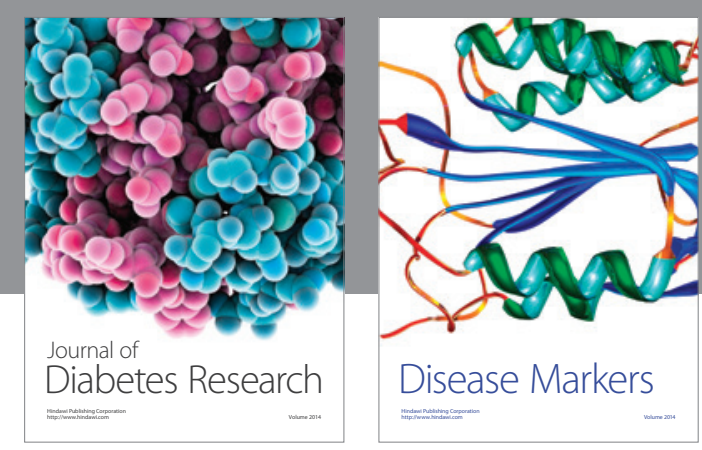

Disease Markers
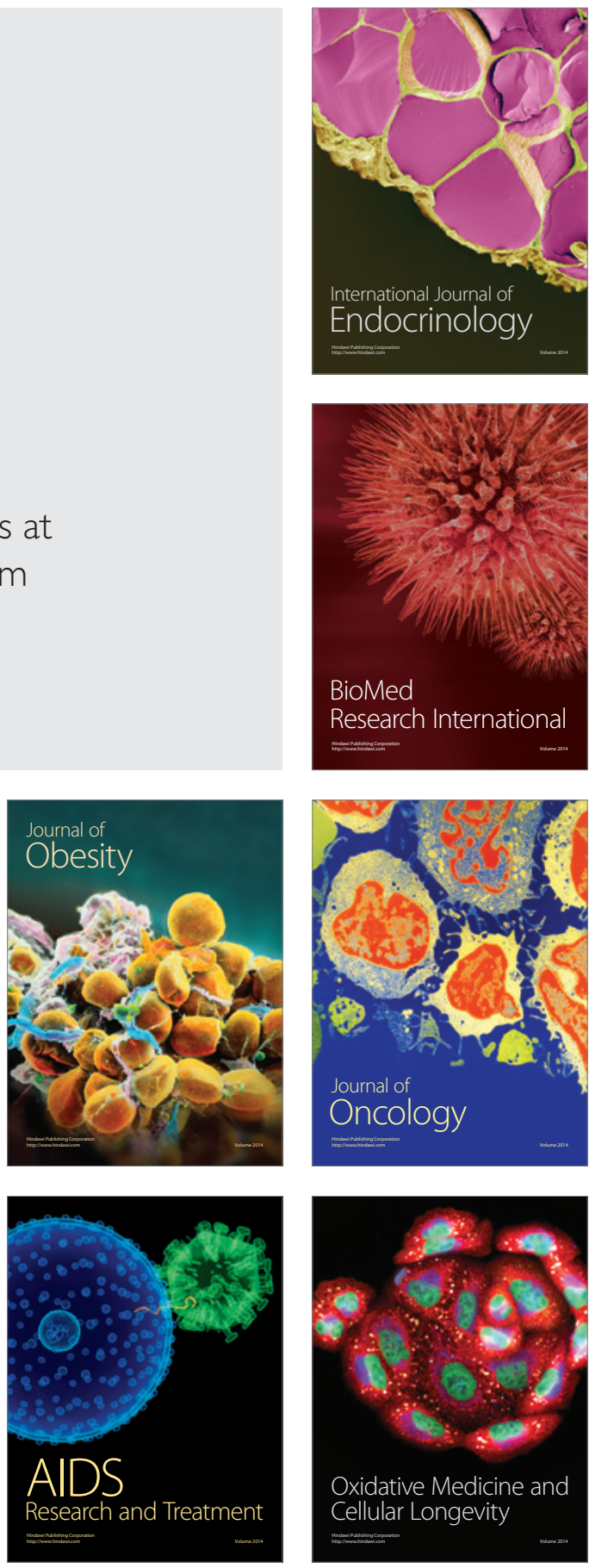